\section{Examination of the Gospel of Judas Using An Integrated Approach to Ink Characterization}

\author{
Joseph G. Barabe, Kathleen A. Martin, \\ Elaine F. Schumacher, \\ Joseph R. Swider, and Anna S. Teetsov \\ McCrone Associates, Inc. \\ Westmont, IL, USA \\ www.mccrone.com
}

\section{Introduction}

In the field of document examination, several approaches can be taken to probe the authenticity of a piece of ancient writing. Scholars have the task of analyzing, and possibly translating, the language and content of a document, and assessing the character and quality of the script. Materials characterization plays another role. Both the document substrate and the ink can be examined to determine whether the materials and methods of creation are consistent with those known to be used during the historical period to which the

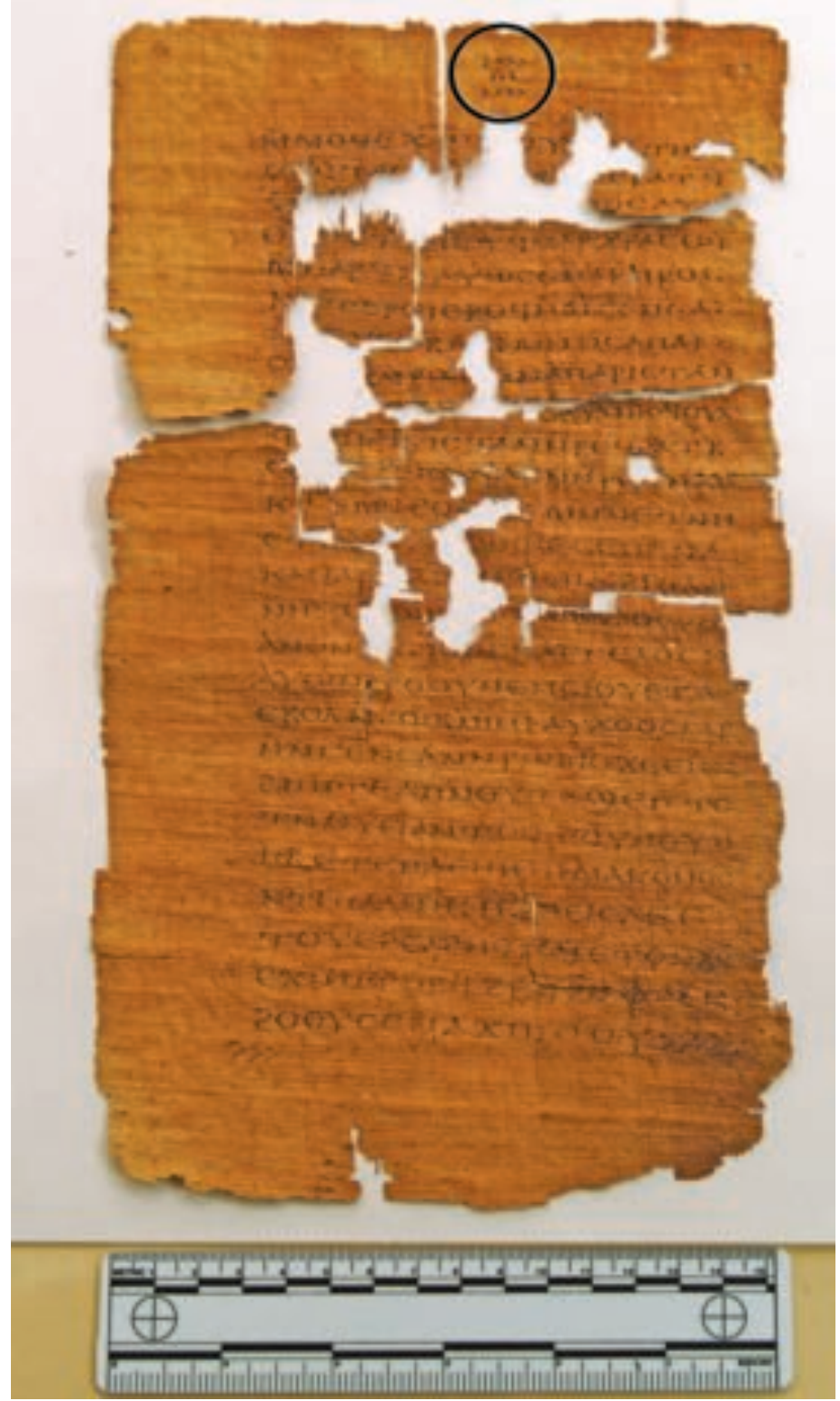

Fig.1. Photograph of codex page 40. Circle indicates location of decorative chevrons from which ink samples were taken. document is attributed. More importantly, the analyst must be on the lookout for the presence of components that were not available during that time, and which would place the document's creation at a later date. Authentication should then really be viewed as 1) failure to uncover evidence of forgery, and 2) placement of results in a known historical context. Provenance is also important; knowledge of how a document was discovered, stored, transported or conserved may aid in accounting for the presence of materials found in the course of analysis.

All of these methodologies were brought to bear on the Gospel of Judas, the discovery and restoration of which was recently revealed by the National Geographic Society. 1 The papyrus document was found in a codex, or ancient book, and is said to date from the third or fourth century C.E. The document is an example of Coptic (Egyptian Christian) writing, and is believed to be a translation of a Greek text written before 180 C.E. The gospel is of tremendous significance to biblical scholars because it portrays Judas Iscariot as a friend to Jesus Christ, rather than a betrayer. It was hidden for nearly 1,700 years before being discovered in the late 1970 's. ${ }^{2}$ The conditions under which it was stored and transported since its discovery have left it in very fragile condition, and it is currently undergoing restoration.

In addition to contextual analysis, the National Geographic Society had the codex materials characterized in several ways. Radiocarbon dating of the papyrus was performed by of Dr. Timothy Jull, Director of the NSF-Arizona Accelerator Mass Spectrometry Laboratory at the University of Arizona, Tucson. The ages of both the papyrus and the leather codex binding fell within the third or fourth centuries C.E. Multispectral imaging of selected pages was performed by Dr. Gene A. Ware, Adjunct Professor at Brigham Young University, Provo, Utah. The work was carried out in the university's Papyrological Imaging Laboratory, and results were consistent with imaging of other ancient papyri examined there. Analysis of the codex ink was performed by a team of scientists at McCrone Associates, Inc. in Westmont, Illinois, where a variety of characterization techniques were used to identify the ink components. The highlights of the ink analysis, performed within a three-month time frame, are presented here.

\section{Document Examination and Sampling}

The team was assembled in early January of 2006 to discuss approaches to sampling and analysis of the document. On 16 January, the document was examined and samples were taken at the studio

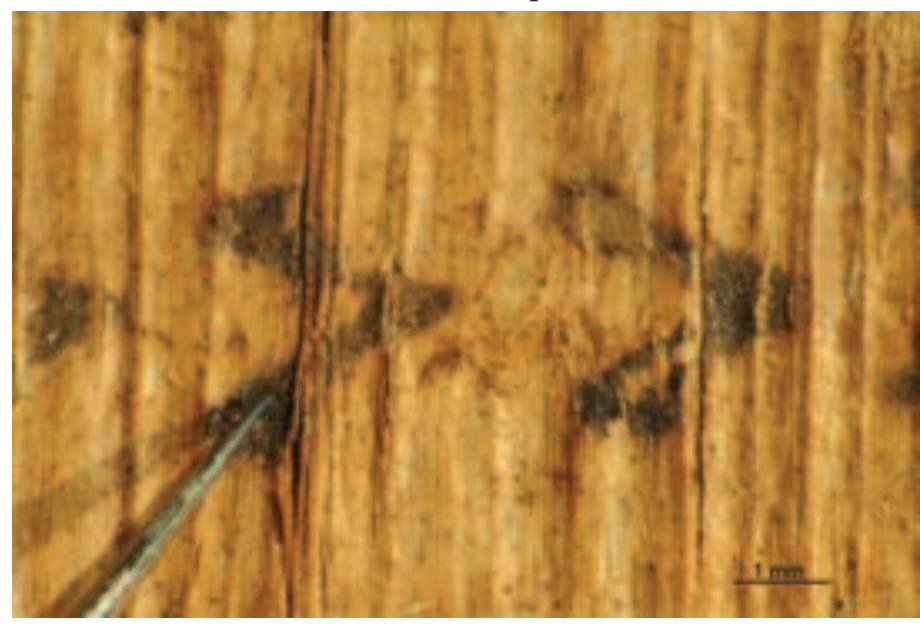

Fig. 2. Optical photomicrograph of ink sampling from decorative chevron using a tungsten needle. 


\section{The Ultimate in Resolution.}

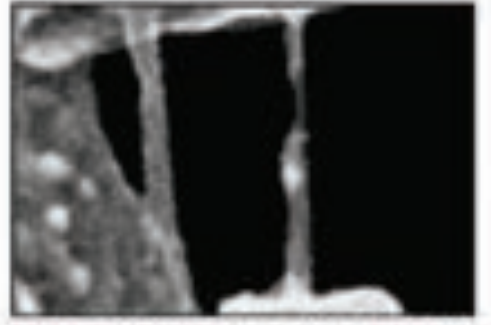

Xic00k, 30 0ar Single Walled Nanotube

The Hitachi S-5500 is a dedicated uttra-high resolution in-lens FE-SEM for leading odge research and dovelopment of nanotechnologies.

The patented in-lens technology provides the ultimate performance of $0.4 \mathrm{~nm}$ guaranteed imaging resclution.

The S- 5500 is equipped with a shielding system for reduced EMI and acoustic interferences. These improvements with a completely dry vacuum system ensures Hitachi's high-resclution guarantee for the ife of the instrument.

A newly designed BFIOF Duo-STEM detector (patent pending) contains an adjustable dark field detector for unabile collection angles.

Sometines a single image can change the way we look at ife. The new S-S500 with its advances in information collection will lead you to those opportunities.

Hitachi High Technologles America, Ine.

S100 Franklis Detve

Plessamen CA 94S88

960.2278877 / exs.218 3230 (f)

mwireactiles con
Hitachi S-5500

In-lens Field Emission Scanning Electron Microscope

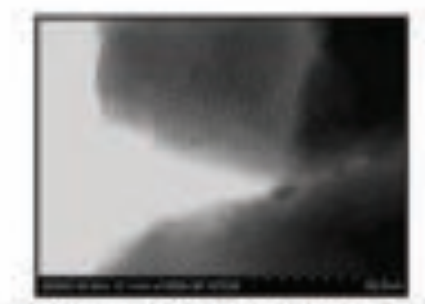

X1000, 30 . ckV Mescoponous Silkea

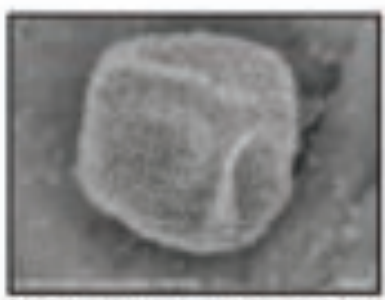

xrock. 5 . ow Immuno Labeling
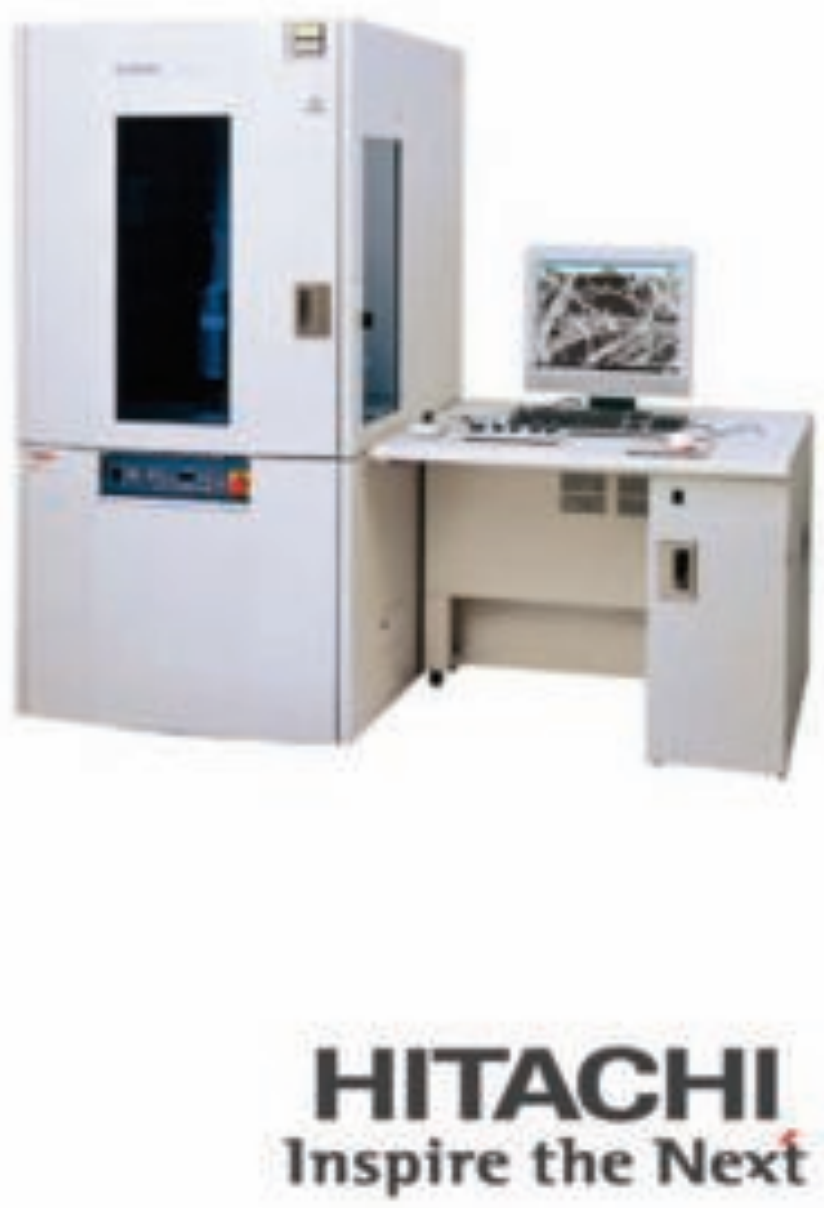

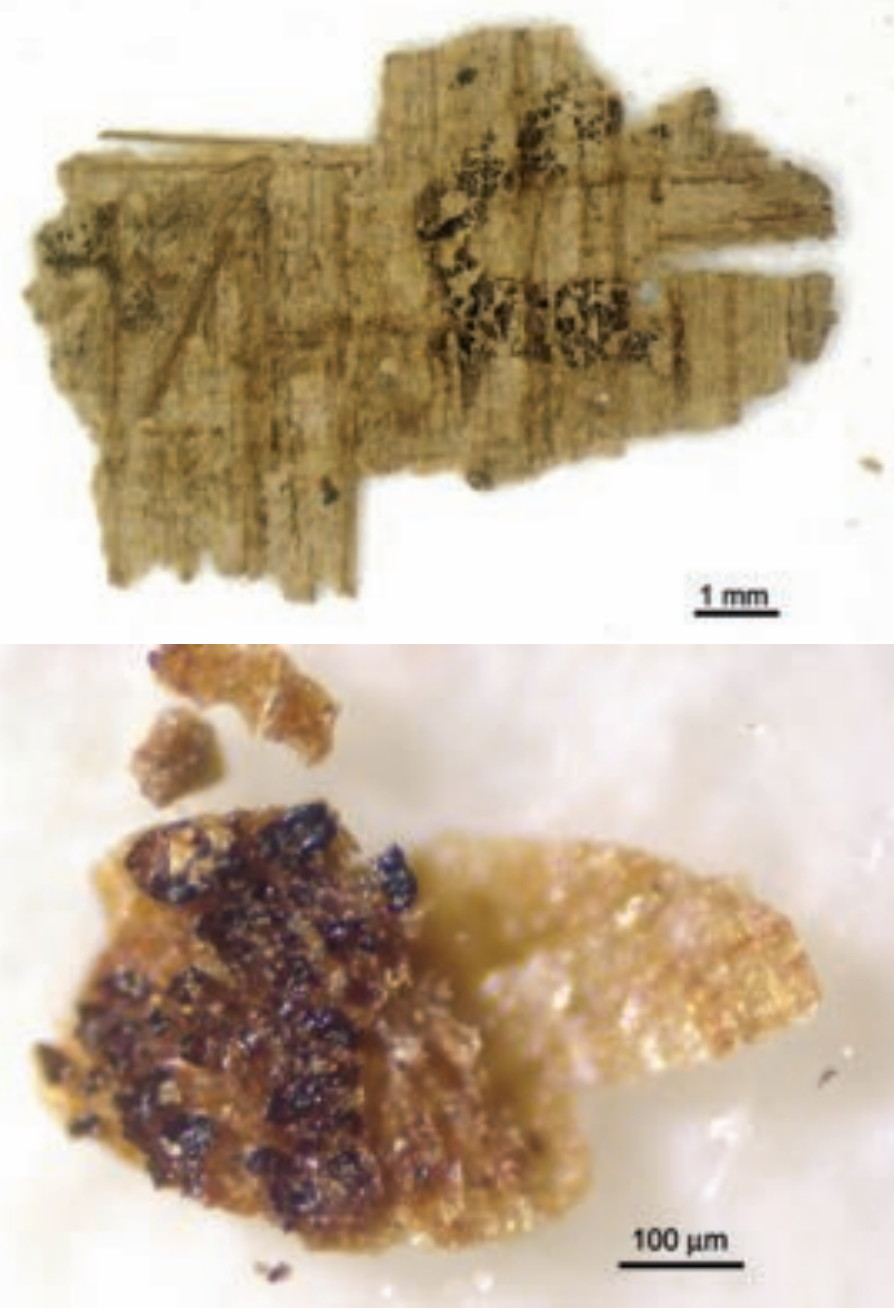

Fig. 3. Optical photomicrographs of papyrus fragments with writing.

of Mme. Florence Darbre, paper conservator, in Cologny (near Geneva), Switzerland.

Two pages of the codex were examined visually and using a stereomicroscope. The pages were severely damaged, but also had large blocks of text intact, as shown in Figure 1, a photograph of codex page 40. An extremely fine-pointed tungsten needle was used to remove small samples of ink from several locations on decorative chevrons at the page tops; Figure 2 illustrates the sampling process. We were also provided with two small papyrus fragments with writing on them, shown in Figure 3. The photomicrographs show the ink to be cracked and grainy, and very loosely attached to the papyrus substrate in many locations.

Samples were prepared for analysis in our Class 100 cleanroom, to avoid possible contamination of the codex materials. Ink and papyrus samples were examined using optical microscopy, and portions were mounted on different substrates for a variety of characterization techniques. Over fifty preparations, ranging from fragments of papyrus to individual ink particles, were mounted and characterized in the course of the project.

\section{Polarized Light Microscopy}

The ink samples were examined using PLM at magnifications between $40 \times$ and $1000 \times$. Most of the material appeared as isotropic, red-brown transparent flakes with conchoidal fracture-characteristics visually consistent with identification as iron gall ink. The particle on the left in Figure 4 is representative. The opaque black particle on the right that appears to be an agglomerate of smaller particles is visually consistent with carbon black. This identification was confirmed using field emission scanning electron microscopy and transmission electron microscopy with energy dispersive $\mathrm{x}$ ray spectrometry. PLM also showed the ink to contain a variety of mineral particles and particles that might originate in plant tissues, such as calcium oxalate.

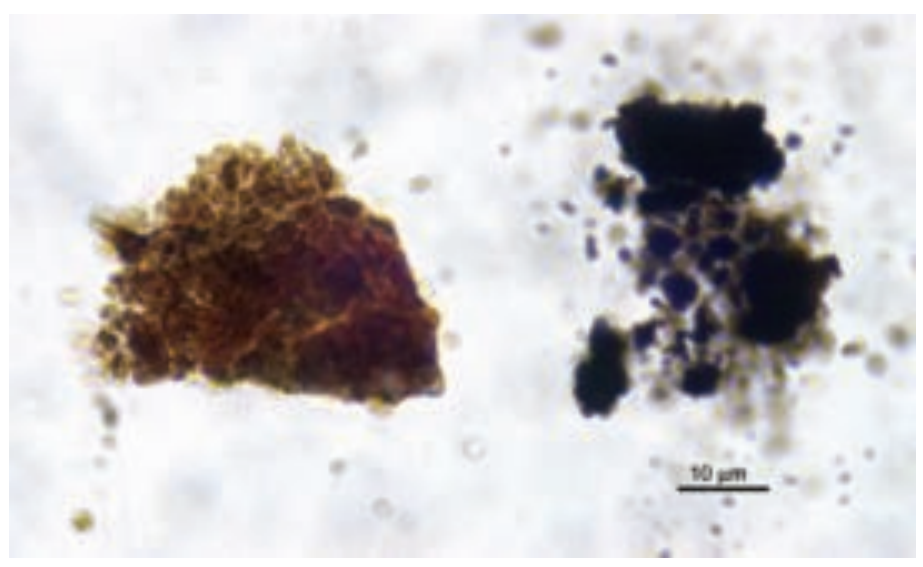

Fig. 4. Optical photomicrograph of ink particles.

\section{Micro Infrared Spectroscopy}

IR spectra from several ink samples were very similar, and the top spectrum shown in Figure 5 is typical. No near matches were found in our library of reference spectra, but the ink spectra are most consistent with the class of gums or other plant resins. Gum Arabic is the material most often cited in early ink-making formulations. Although the IR spectrum of modern gum Arabic (Figure 5 bottom spectrum) does not closely match the spectrum of the ink, it shows the broad bands typical of complex natural materials. The relative intensities and positions of the bands depend upon both the composition and degree of oxidation of the material.

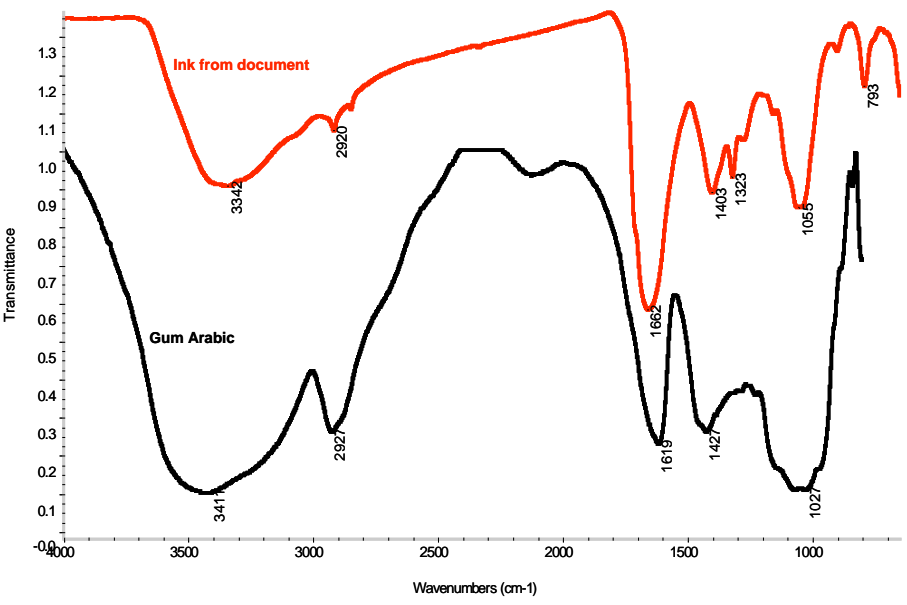

Fig. 5. IR spectrum of ink from codex document (top) and reference IR spectrum of gum Arabic (bottom).

\section{Raman Spectroscopy}

Most Raman spectra collected from ink particles and document fragments matched amorphous carbon, as shown in Figure 6. However, some spectra were suggestive of iron gall ink. The stacked plot in Figure 7 shows a spectrum from the codex ink (top) and a fresh iron gall ink (bottom). Shifts in some of the peaks may result from oxidation of the iron species in the codex ink with aging. This is supported by the peak shifts seen in the spectrum from a fresh iron 


\section{When Light Microscopy simply isn't enough!}

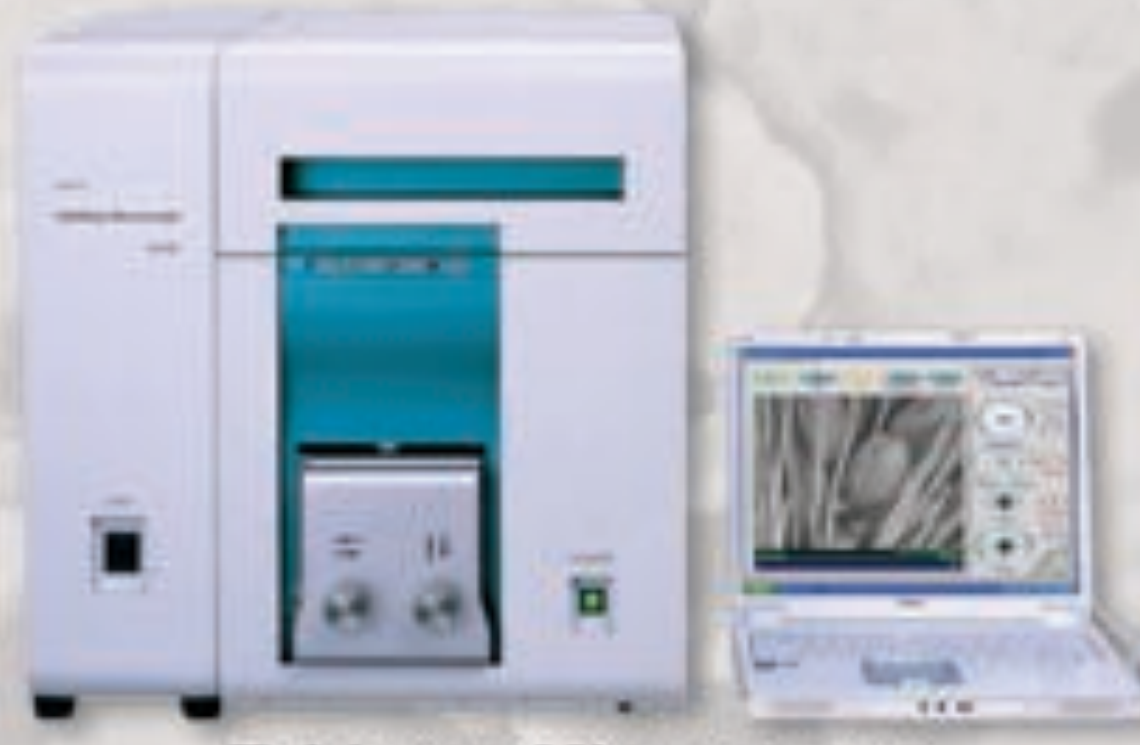

\section{Tabletop Microscope TM-1000}

No coating required. 20-10,000 x magnification with $25 \mathrm{~nm}$ resolution. As easy to use as a digital camera with easy maintenance. Stereoscopic observation with greater depth of focus.

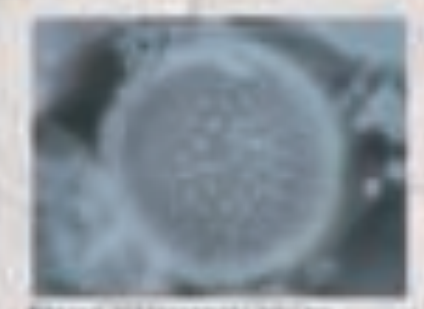

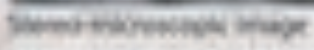

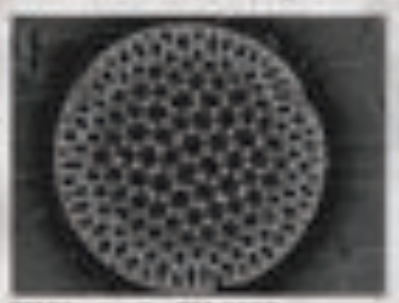

sivivage by Tu. 1000

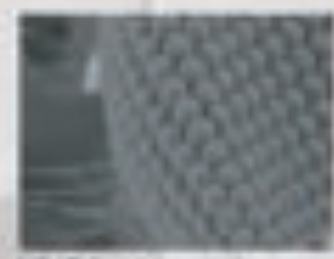

LiR Solerse

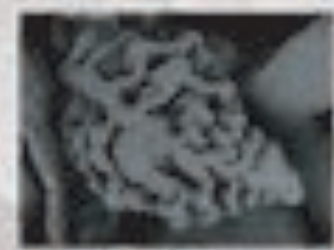

Nologed

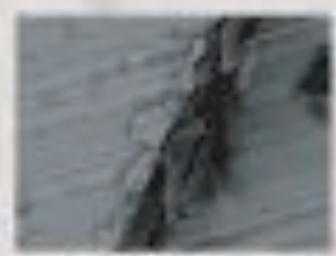

Manifar Scleres

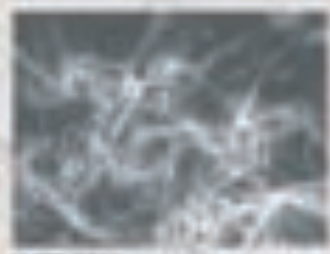

WTREM

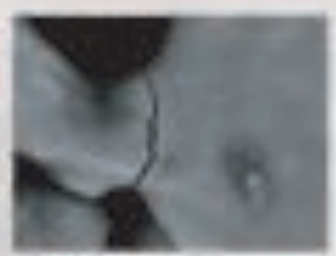

follenowisis

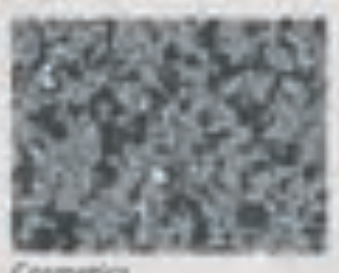

Coumetica

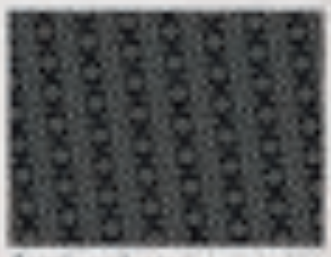

Sevilicianducter

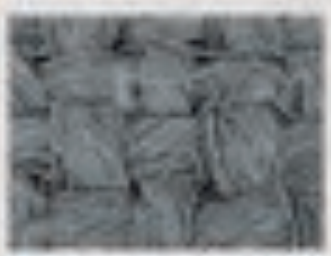

Thesken 
gall ink aged artificially using a laser (center); the resulting spectrum is more similar to that of the codex ink sample.

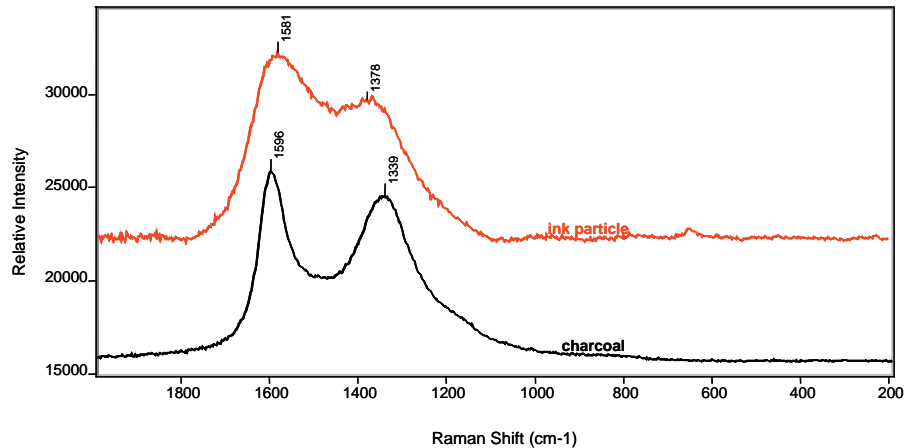

Fig. 6. Raman spectrum of ink particle from codex document (top) and reference Raman spectrum of charcoal (amorphous carbon, bottom).

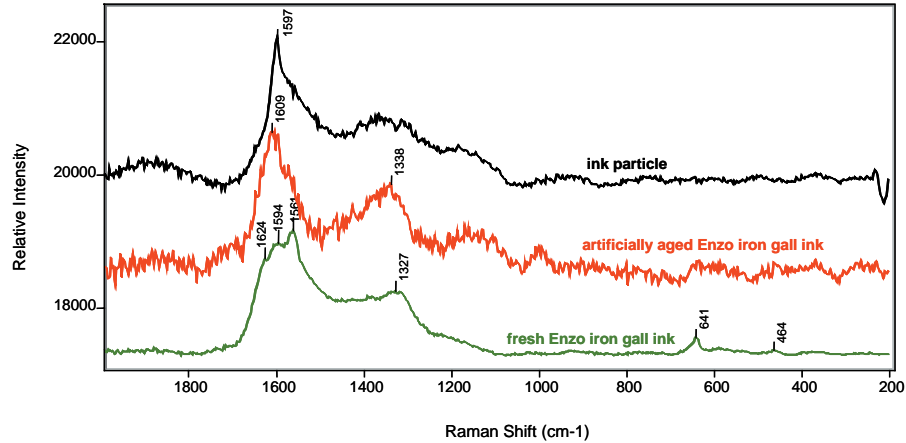

Fig. 7. Raman spectra of ink sample from codex (top), fresh iron gall ink (bottom) and artificially aged iron gall ink (center). Note shift in peaks from fresh to aged ink.

\section{Electron Microscopy - Carbon Ink}

Material optically characterized as black ink, and suspected to contain soot in the form of carbon black, was analyzed using FE-SEM imaging and TEM-EDS. The FE-SEM image in Figure 8 shows the black ink particles to have the aciniform, or 'grape-cluster' morphology characteristic of carbon black aggregates. Figure 9 (top) is a TEM image of a black ink aggregate, and Figure 9 (bottom) is a

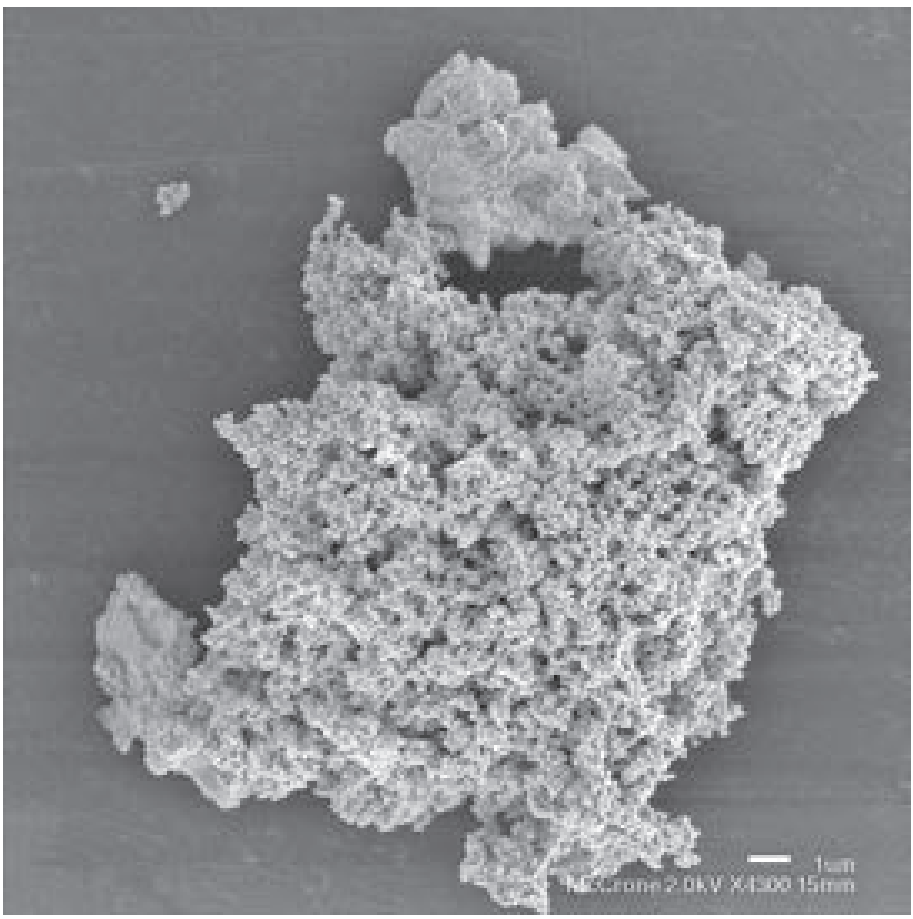

Fig. 8. FE-SEM image of carbon ink particle. corresponding EDS spectrum. The aggregate is comprised of amorphous, roughly rounded particles containing mainly carbon.

The carbon ink was present on the document in a much lower proportion than the iron tannate ink described below. Iron tannates begin as gray materials, eventually turning deep black through oxidation of the iron. Such ink formulas typically include a temporary colorant to provide depth until the blackening occurs. Carbon black has been fabricated for over two millennia for use as an ink, and could have been added to the codex formulation to achieve this end. ${ }^{3}$ It is possible that the document originally contained a higher proportion of carbon ink, but that much of it has flaked away over time.

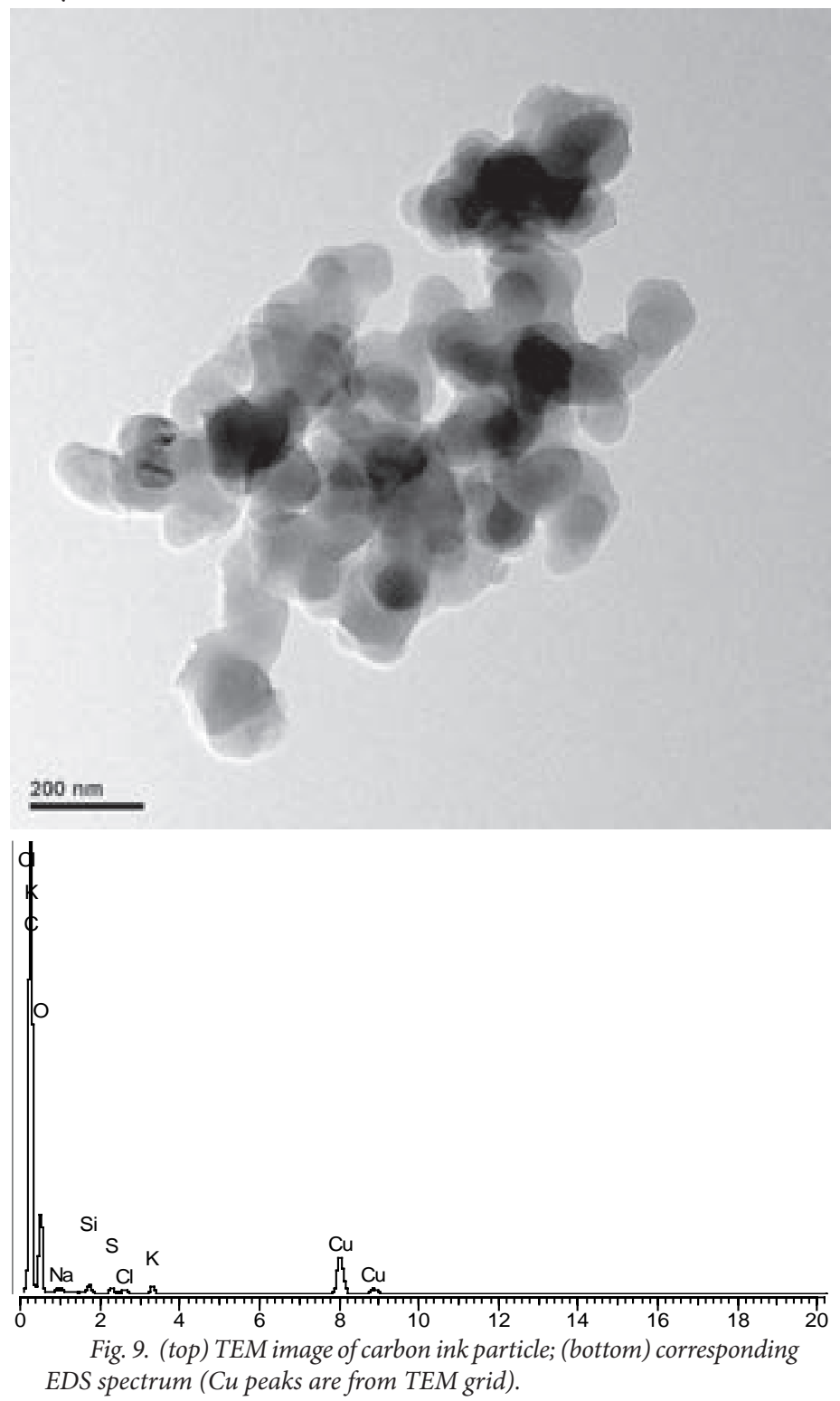

\section{Electron Microscopy - Iron Tannate Ink}

The majority of the ink particles sampled did not appear to be carbon black, and extensive SEM and TEM imaging and EDS analysis showed the ink to have an iron-rich component. These particles tended to be angular fragments with irregular shapes and a wide size distribution. A TEM image of a typical aggregate of particles is shown in Figure 10, and FE-SEM images of particles are shown in Figure 11. Some exhibited quite a bit of fine surface particulate, as can be seen in the bottom image. 
- and CHotedi has souething ane to shor you

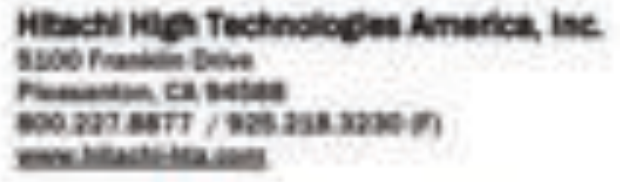

Vhit Bocth 1420 ef sesk zoob
HITACHI

Inspire the Next 


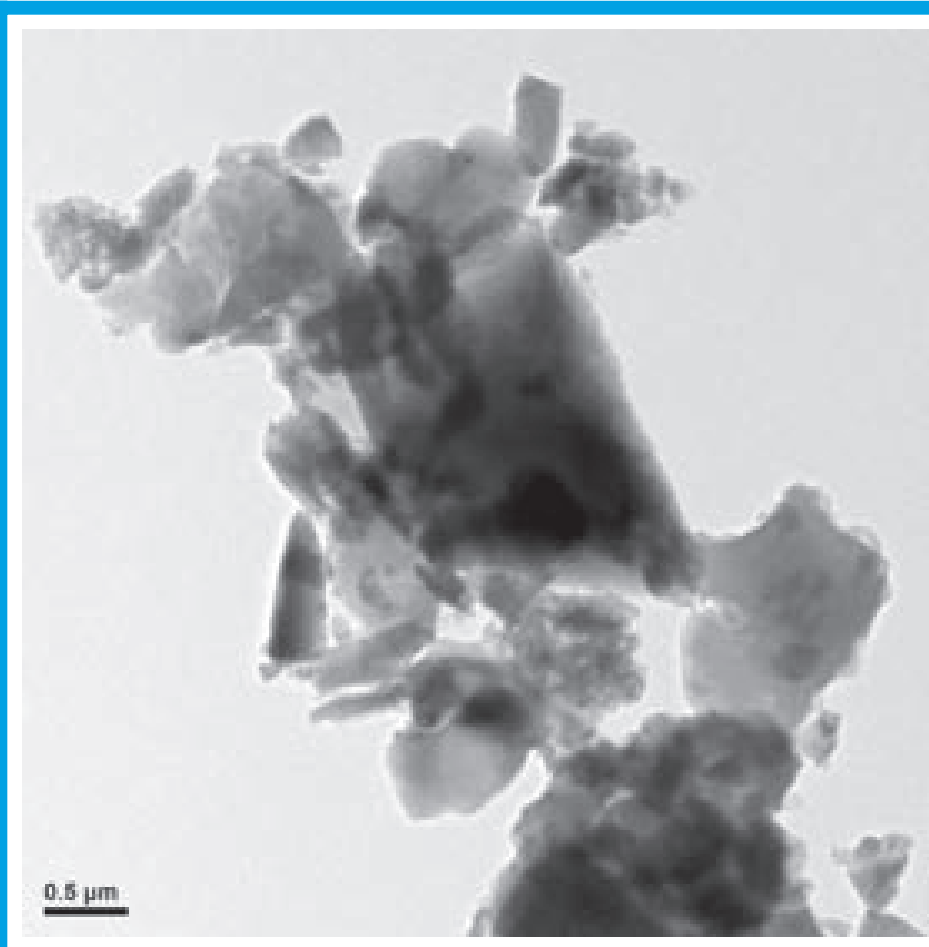

Fig. 10. TEM image of aggregate of typical iron-rich ink particles.

A representative SEM-EDS spectrum of the iron-rich ink particles is shown in Figure 12. This spectrum was acquired from a highly homogeneous particle with no evidence of inclusions in backscattered electron imaging mode. Such particles tended to contain major amounts of iron, potassium and phosphorus in atomic ratios of roughly 3:2:1, with minor amounts of sodium, chlorine and sulfur, and highly variable amounts of calcium, silicon, and magnesium/aluminum/silicon in proportions suggesting clay.

TEM-EDS analysis at higher spatial resolution supported initial SEM findings for the iron ink chemistry, and clarified the association of elements present. The spectra shown in Figure 13 illustrate the particle types that comprised the majority of the material analyzed. Spectrum A is representative of typical isolated ink particles, and shows the Fe/K/P elemental profile described above. Spectrum B was acquired from a crystalline particle with a plate-like morphology, and the $\mathrm{Mg} / \mathrm{Al} / \mathrm{Si} / \mathrm{O}$ composition is consistent with identification of this as a clay, probably of environmental origin. Spectrum $\mathrm{C}$ consists mainly of calcium and oxygen, indicating presence of calcium oxide or calcium oxalate, crystals of which are commonly produced by many plants.

The presence of iron would suggest a typical iron gall ink, made from extracts of galls from oak trees (a source of tannin) mixed with copperas (iron sulfate). The EDS spectrum of a traditional iron gall ink includes both iron and sulfur, in roughly equal amounts. The absence of any but trace amounts of sulfur in the codex ink indicates that the ink was made from some other formula, or that the sulfur may have been separated from the ink or from the document by an unknown mechanism.

The presence of significant amounts of potassium, phosphorus, calcium and sodium associated with the ink particles suggest a possible plant-based ink formulation, as all of these elements are found in plants. They were also found in analysis of the papyrus substrate and a modern papyrus, as described below. However, the large amounts of these elements present in the ink particles make it more likely that they were present in the ink formulation, rather than leaching into the wet ink when it was applied to the papyrus.
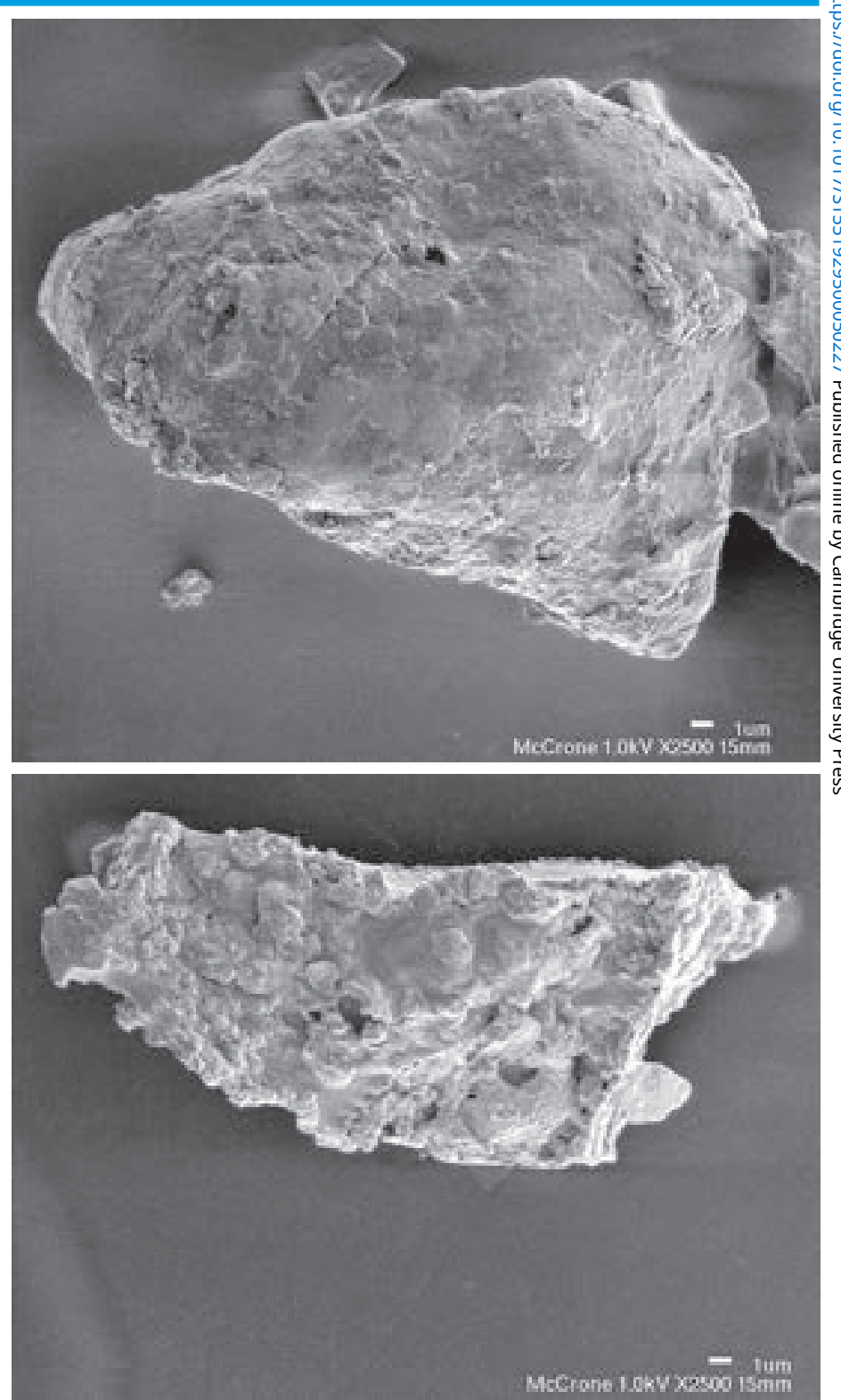

Fig. 11. FE-SEM images of typical iron-rich ink particles.

Wine lees (dregs), walnut husks and many types of tree barks are mentioned in the literature as possible sources of tannins incorporated into ink formulations. Another possible source is pomegranate

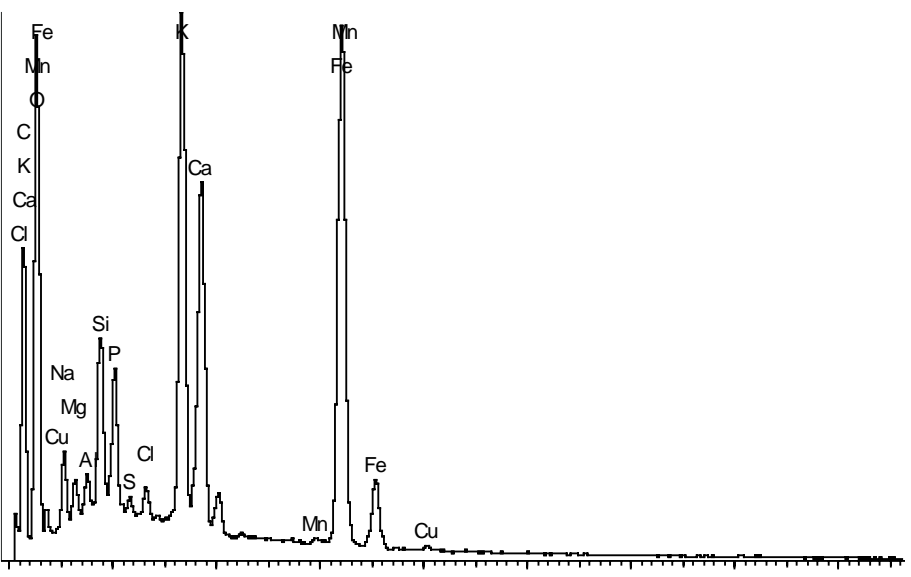

Fullscale B776 ots Culsos:-0.176 lo ols

Fig. 12. Representative SEM-EDS spectrum of iron-rich ink particles. 


\section{닝}

ORIUS

11 MEGAPIXEL FIBER-OPTICALLY COUPLED HIGH SPEED CCD CAMERA

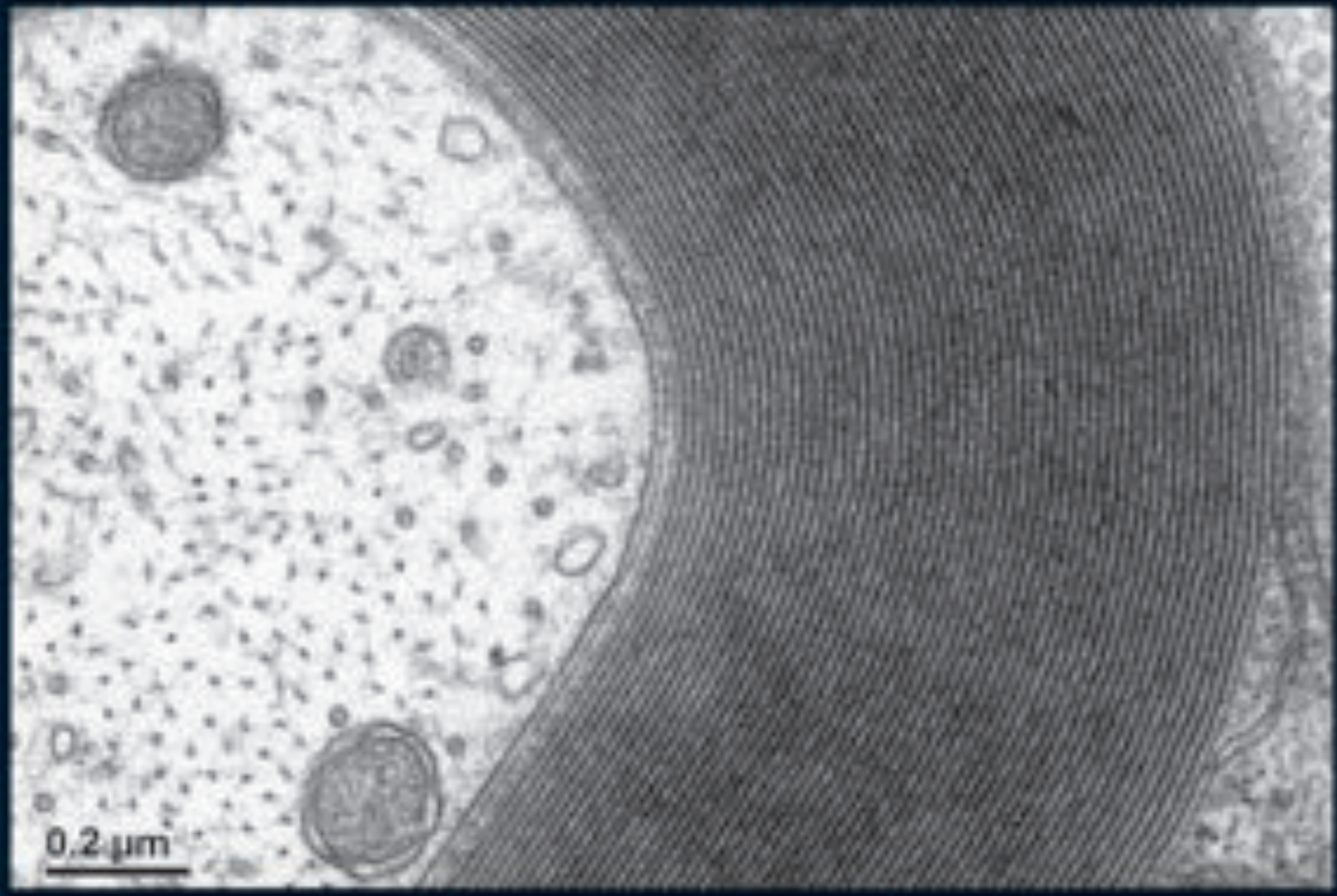

AEAL-TIME VIEW

HIOH-REgLLTION IMAOINB

LAAGE FIELD OF VIEW RECORDING DIFFAACTION PATTEANS

DIGITAL GTREAMINE VIDEO

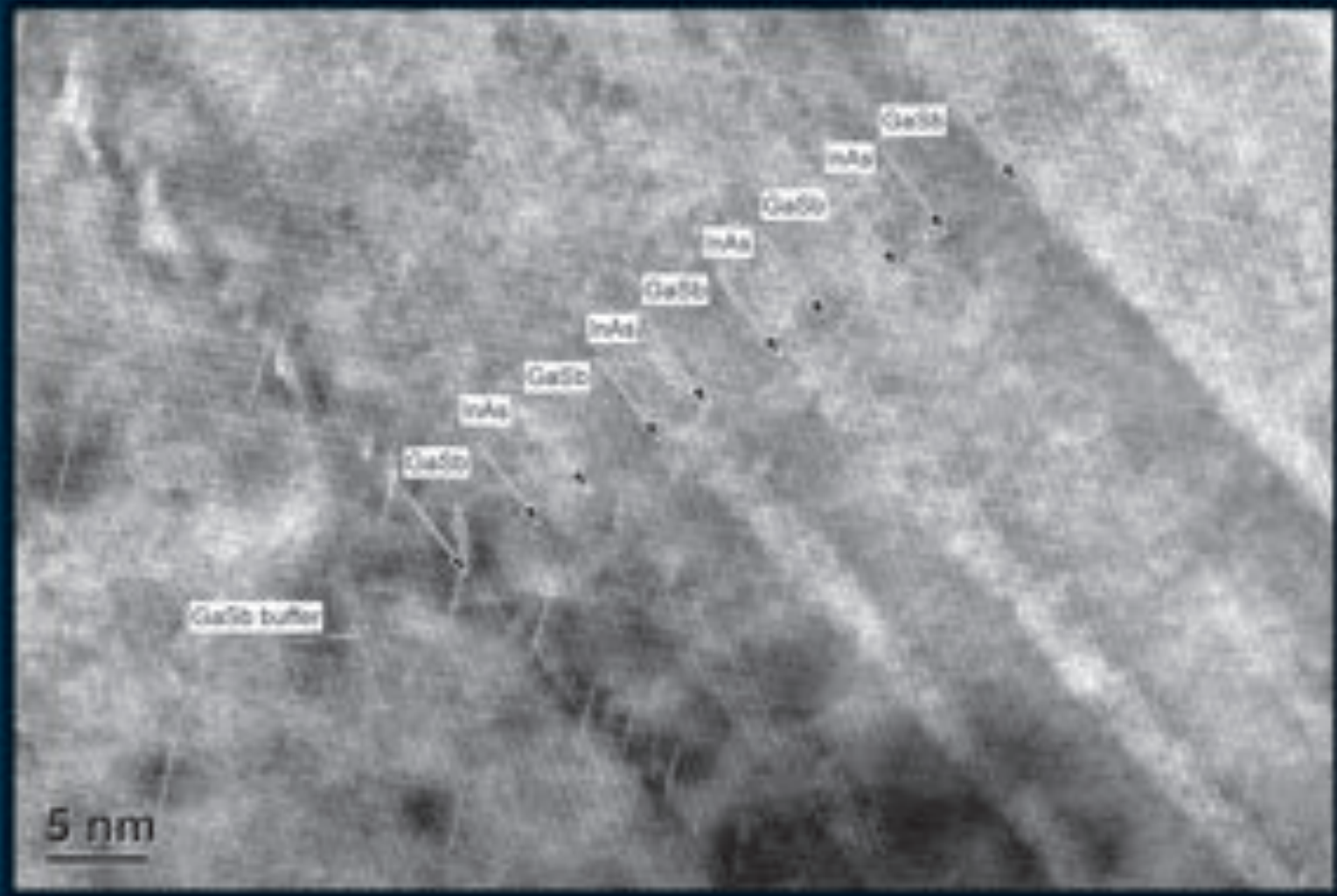




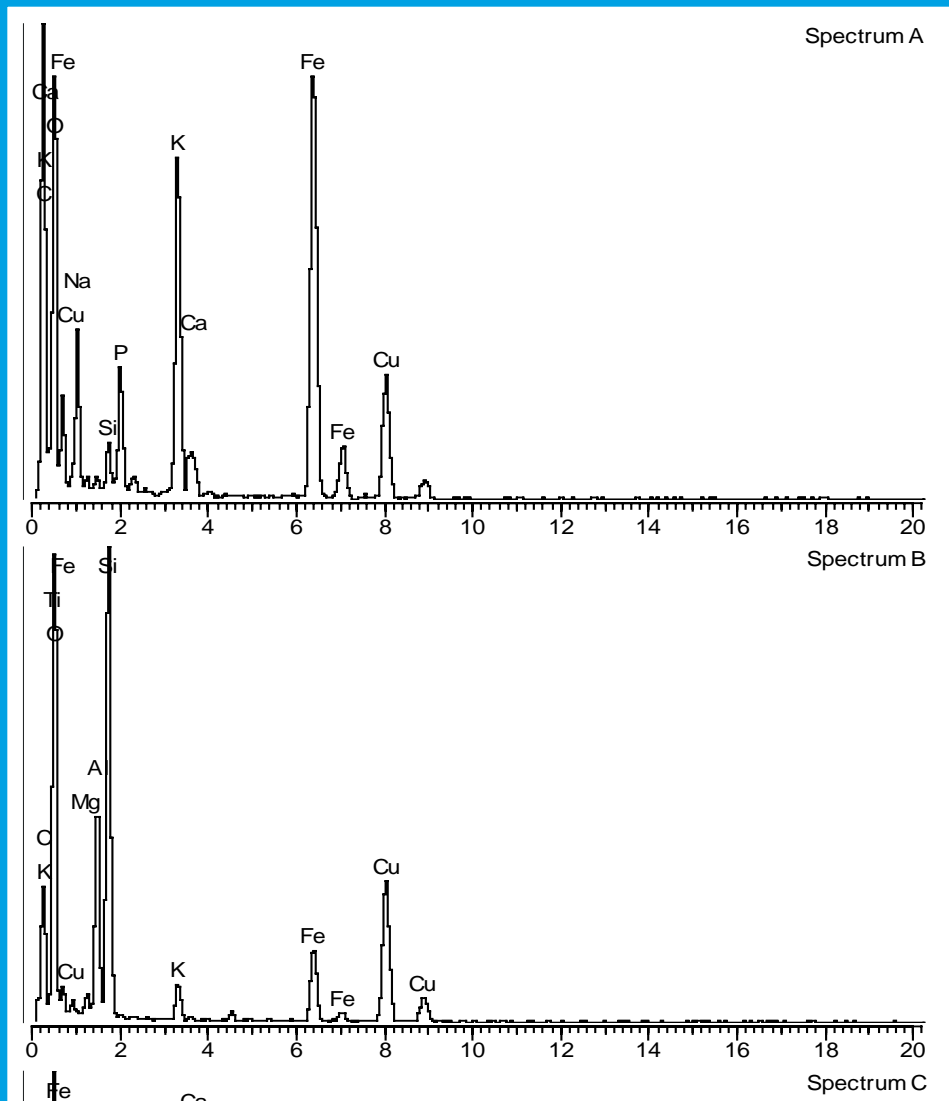

mediately adjacent to ink lines and in areas away from the ink lines. Samples were also taken from the document surface and from layers beneath the surface. Additionally, samples of both the old and new papyrus were subjected to low temperature ashing to concentrate the inorganic elements. SEM-EDS was used to analyze the various papyrus samples.

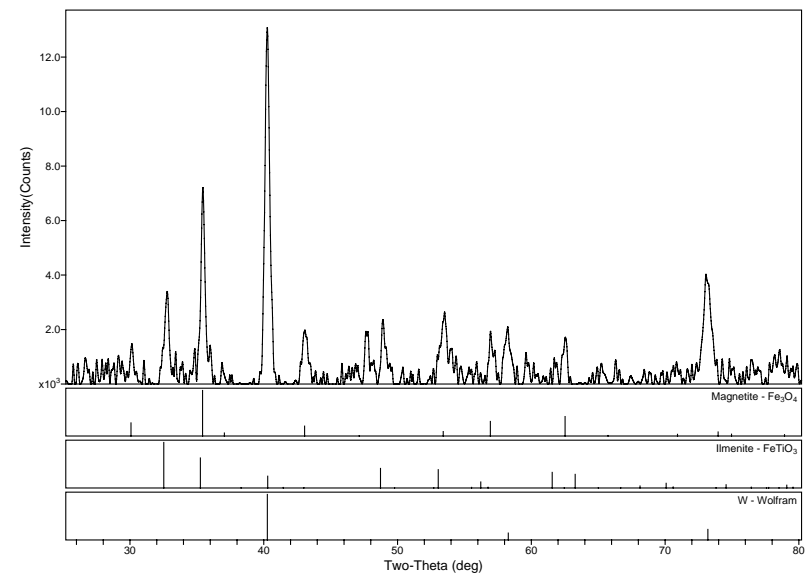

Fig. 14. XRD pattern of particle rich in iron and titanium, and reference stick patterns of magnetite and ilmenite. Some peaks match tungsten, likely from sampling needle.

Though rich in carbon, as would be expected, the non-inked areas of the papyrus were found to contain small amounts of iron and other elements associated with the ink formulation, indicating the probable presence of ink particles in areas away from the ink lines. This is consistent with optical microscopy observations of the cracked and flaking texture of the ink, and of fine particles scattered on non-inked areas. The old and new papyrus samples were also found to contain varying amounts of potassium, calcium, sodium, silicon and phosphorus.

\section{Conclusions}

Samples of ink and papyrus associated with the document known as the Gospel of Judas were examined using PLM, IR and Raman spectroscopies, SEM and TEM imaging and EDS, and XRD. The combined results support several conclusions about the nature of the ink:

Fig. 13. TEM-EDS spectra of representative particle types: (A) Fe/K/P ink, (B) aluminosilicate, (C) probable calcium oxide or oxalate (Cu peaks are from TEM grid).

rind, which fits the chemical data well, and is frequently mentioned in the literature. ${ }^{4}$ Some ink formulas describe boiling the rind in a hot alkali solution, which would also provide a source for the potassium, sodium, calcium and phosphorus found in the codex ink.

\section{Micro X-Ray Diffraction}

Micro XRD indicated that the majority of the ink particles were amorphous, but two crystalline mineral phases were identified. Particles found to contain both iron and titanium by SEM-EDS were subsequently mounted on a glass fiber for XRD analysis. The particle pattern and reference patterns shown in Figure 14 indicate the presence of magnetite, $\mathrm{Fe}_{3} \mathrm{O}_{4}$, and ilmenite, $\mathrm{FeTiO}_{3}$, both of which are naturally occurring minerals. These results are consistent with PLM identification of mineral particles in the ink samples, and with TEM observations.

\section{Analysis of Papyrus}

Samples of the codex papyrus and of a modern day papyrus were analyzed to estimate the contribution of the papyrus substrate to the ink elemental profile. The codex papyrus was sampled imtannate. However, it does not seem to have been manufactured by a traditional iron gall formula, which would include a significant amount of sulfur. The ink particles primarily contain iron, potassium and phosphorus in atomic ratios of roughly 3:2:1. Sodium is also often associated with the ink particles. The ink formula may have been plant based, and the chemistry is consistent with literature descriptions of formulas incorporating pomegranate rind.

- Calcium is frequently present, usually as discrete particles, possibly calcium oxide or calcium oxalate. This is most likely of plant origin.

- A carbon black pigment was found in minor amounts, primarily at the surface of the ink. It was seen to be composed of very fine particles consistent with lampblack or soot black pigment. This may have been applied as a darkening agent, and much of it may have flaked off over time.

- Most particles in the ink were found to be amorphous, but plate-like particles of crystalline magnesium aluminum silicate were observed, and are most likely clay; this could have an environmental origin, such as a pottery vessel for mixing or storage 
of ink. Both crystalline and amorphous silica were found; the crystalline material is probably quartz of environmental origin, while amorphous silica may be associated with plant materials. Particles of magnetite and ilmenite were also identified.

- The carrier is most likely a resin or gum, possibly gum Arabic.

The results produced by our team of analysts are consistent both internally and with results obtained by other laboratories using complementary techniques. So far, the chemical composition of the codex ink has not been found to match that of any modern ink, and the materials identified are those that would be expected for a third century C.E. document found in Egypt. There is nothing in our findings to suggest that the document is not authentic. Examination and analysis of papyrus documents of a similar age and of known provenance would aid in the interpretation of results from the codex samples.

Compared to ink and document analyses previously performed at McCrone Associates, the Gospel of Judas presented some challenges, not the least of which was the complex, multi-component nature of the ink. The iron component in particular did not readily fit known ink formulations of the time, such as iron gall ink. Integrating results from a variety of techniques allowed us to characterize both organic and inorganic constituents of the ink and substrate for morphology, crystallinity and elemental and molecular composition, and the combined efforts of a team of analysts ensured that the work could be completed in the short time frame allowed.

\section{Acknowledgements}

We would like to thank the National Geographic Society for granting their permission to publish this work.

\section{References}

1. news.nationalgeographic.com, follow links to History and Culture News, and Archeology and Paleontology News.

2. Kasser, R., Meyer, M., Wurst, G., The Gospel of Judas, National Geographic, Washington D.C. (2006).

3. Swider, J.R., Hackley, V.A., Winter, J., Characterization of Chinese ink in size and surface, Journal of Cultural Heritage 4 (2003) 175-186.

4. Carvalho, D.N., Forty Centuries of Ink, New York, 1904, pp. 63-81.
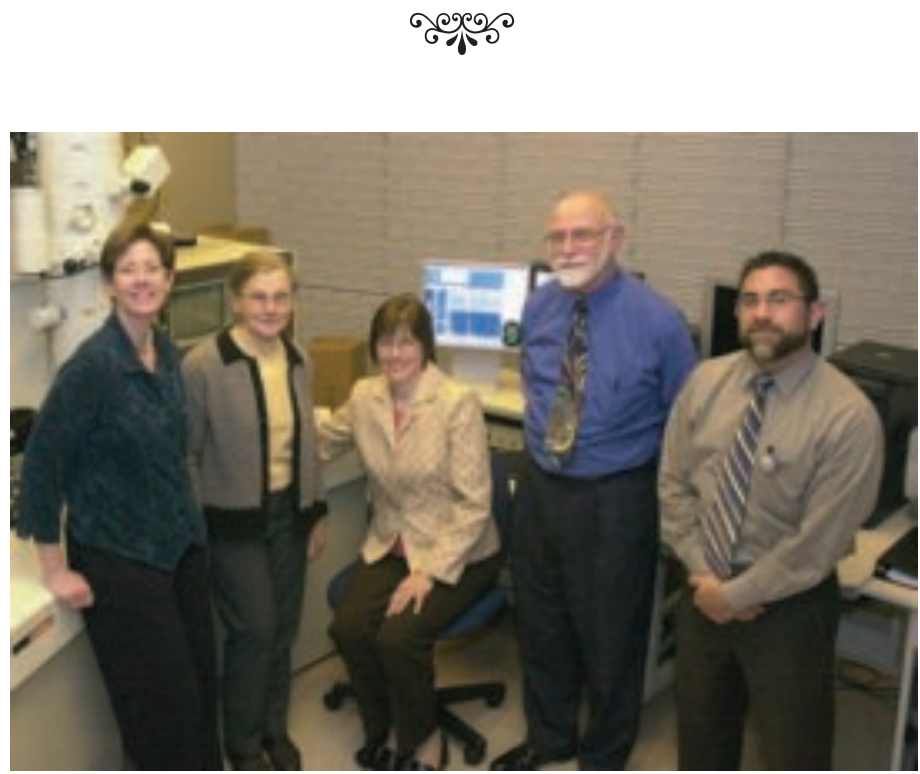

McCrone Associates Gospel of Judas Team (left to right): Dr. Kathleen Martin - IR and Raman spectroscopy, Anna Teetsov - Sample preparation, Elaine Schumacher - TEM, Joseph Barabe - Team leader, document examination and sampling, and Dr. Joseph Swider-SEM, XRD. (Photo courtesy of Mr. Bill Ackerman, Liberty Suburban Chicago Newspapers)

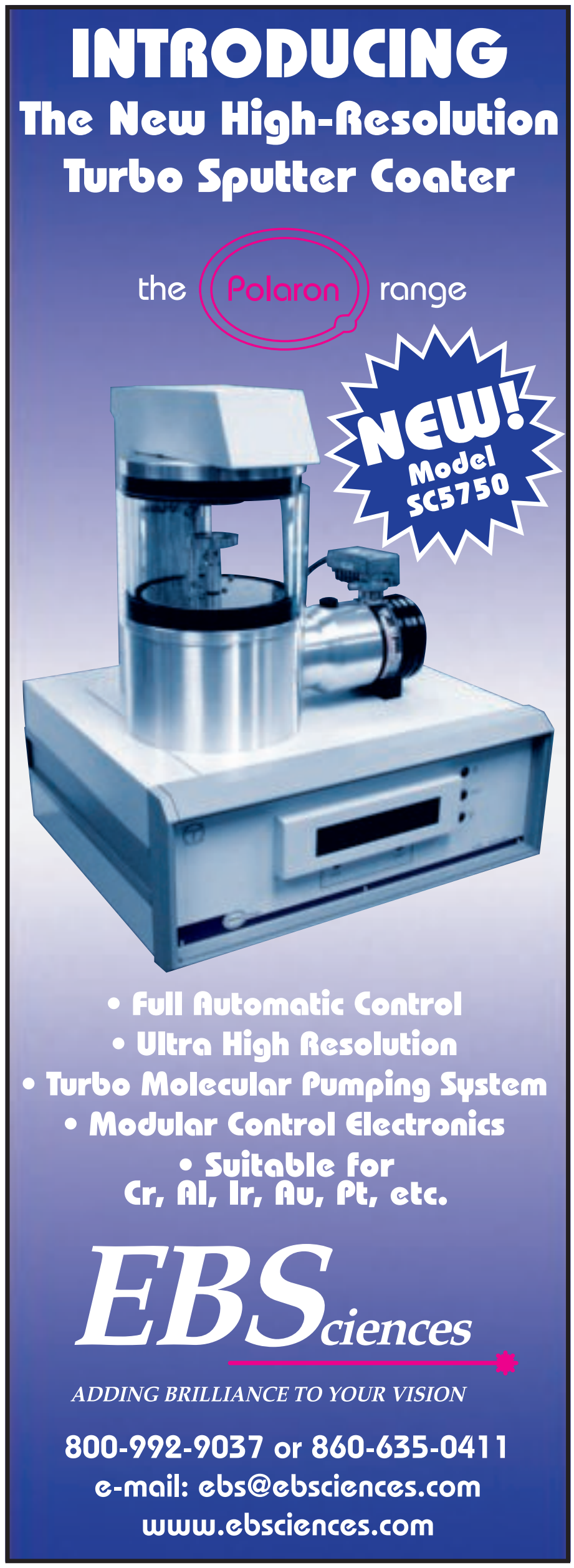

mICROSCOPY TODAY July 2006 